\title{
SM and MSSM Higgs Boson Production: Spectra at large transverse Momentum
}

\author{
Urs Langenegger ${ }^{1}$, Michael Spira ${ }^{2}$, Andrey Starodumov ${ }^{1}$, and Peter Trüb ${ }^{1,2}$ \\ ${ }^{1}$ Institute for Particle Physics, ETH Zurich, 8093 Zurich, Switzerland \\ ${ }^{2}$ Paul Scherrer Institute, 5232 Villigen PSI, Switzerland
}

(Dated: June 4, 2018)

\begin{abstract}
Strategies for Higgs boson searches require the knowledge of the total production cross section and the transverse momentum spectrum. The large transverse momentum spectrum of the Higgs boson produced in gluon fusion can be quite different in the Standard Model and the Minimal Supersymmetric Standard Model. In this paper we present a comparison of the Higgs transverse momentum spectrum obtained using the PYTHIA event generator and the HIGLU program as well as the program HQT, which includes NLO corrections and a soft gluon resummation for the region of small transverse momenta. While the shapes of the spectra are similar for the Standard Model, significant differences are observed in the spectra of Minimal Supersymmetric Standard Model benchmark scenarios with large $\tan \beta$.
\end{abstract}

PACS numbers: $14.80 . \mathrm{Bn}, 14.80 . \mathrm{Cp}, 12.60 . \mathrm{Jv}$

\section{INTRODUCTION}

The search for Higgs bosons belongs to the most important endeavors at the Large Hadron Collider (LHC) in order to establish experimentally the Higgs mechanism for electroweak symmetry breaking. In the Standard Model (SM) one isospin Higgs doublet is introduced, which leads to the existence of one physical Higgs particle after electroweak symmetry breaking, while the other three degrees of freedom are absorbed by the $W$ and $Z$ bosons [1]. In the SM Higgs sector the only unknown parameter is the Higgs mass. Based on triviality and unitarity arguments its value is required to range below $\sim 800 \mathrm{GeV}$ [2, 3]. The Higgs couplings to fermions and electroweak gauge bosons grow with the corresponding masses $(V=W, Z)$

$$
g_{f f h}^{S M}=\left(\sqrt{2} G_{F}\right)^{1 / 2} m_{f}, \quad g_{V V h}^{S M}=\left(\sqrt{2} G_{F}\right)^{1 / 2} m_{V}^{2},
$$

where $G_{F}=1.16637 \times 10^{-5} \mathrm{GeV}^{-2}$ is the Fermi constant. Therefore, the Higgs couplings to the $W$ and $Z$ bosons as well as to third-generation fermions are phenomenologically relevant, while the couplings to the first two generations are less important. The direct search at the LEP2 experiments excluded Higgs masses below $114.4 \mathrm{GeV}$ [4]. If the SM is embedded in a Grand Unified Theory (GUT), the quadratically divergent radiative corrections to the Higgs selfenergy tend to push the Higgs mass towards the GUT scale $M_{\mathrm{GUT}} \sim 10^{16} \mathrm{GeV}$. In order to establish a Higgs mass of the order of the electroweak scale an unnatural fine-tuning of the counter terms is required. This hierarchy problem remains unsolved within the SM.

The most attractive solution to the hierarchy problem is the introduction of supersymmetry (SUSY), a novel symmetry between fermionic and bosonic degrees of freedom [5]. Due to the additional contributions of the SUSY partners of each SM particle the quadratic divergences in the Higgs selfenergy are canceled. The hierarchy problem is solved [6], if the SUSY particle masses are maximally of the order of a few TeV. In the minimal supersymmetric extension of the SM (MSSM) two isospin Higgs doublets have to be introduced in order to preserve SUSY [7] and to render the model
TABLE I: Higgs couplings in the MSSM to fermions and gauge bosons $(V=W, Z)$ relative to the SM couplings.

\begin{tabular}{|c|c|c|c|c|}
\hline \multicolumn{2}{|l|}{$\phi$} & $g_{u}^{\phi}$ & $g_{d}^{\phi}$ & $g_{V}^{\phi}$ \\
\hline SM & $h$ & 1 & 1 & 1 \\
\hline MSSM & $H$ & $\begin{array}{c}\cos \alpha / \sin \beta \\
\sin \alpha / \sin \beta \\
1 / \tan \beta\end{array}$ & $\begin{array}{c}-\sin \alpha / \cos \\
\cos \alpha / \cos \beta \\
\tan \beta\end{array}$ & $\begin{array}{c}\sin (\beta-\alpha) \\
\cos (\beta-\alpha) \\
0\end{array}$ \\
\hline
\end{tabular}

free of anomalies. After electroweak symmetry breaking five Higgs bosons are left as physical particles: two $\mathcal{C P}$-even neutral (scalar) particles $h, H$, one $\mathcal{C P}$-odd neutral (pseudoscalar) particle $A$ and two charged bosons $H^{ \pm}$.

At leading order the Higgs sector is determined by two independent input parameters, which are usually chosen as the pseudoscalar Higgs mass $M_{A}$ and $\tan \beta=v_{2} / v_{1}$, the ratio of the two vacuum expectation values. The light scalar Higgs boson $h$ has to be lighter than the $Z$ boson at leading order. This upper bound, however, is significantly enhanced to a value of $\sim 140 \mathrm{GeV}$ due to radiative corrections, which are dominated by top- and stop-loop contributions [8, 9]. Moreover, all Higgs couplings are affected by the same type of corrections. The couplings of the Higgs bosons to fermions and gauge bosons are modified by coefficients, which depend on the angles $\alpha$ and $\beta$, where $\alpha$ denotes the mixing angle of the two $\mathcal{C} \mathcal{P}$-even Higgs fields. The couplings, normalized to the SM Higgs coupling, are listed in Table@ An important property of these couplings is that for large values of $\tan \beta$ the down(up)-type Yukawa couplings are strongly enhanced (suppressed). The direct Higgs searches at the LEP2 experiments have excluded neutral Higgs masses $M_{h, H} \lesssim 91.9 \mathrm{GeV}$ and $M_{A} \lesssim 91.0 \mathrm{GeV}$ as well as charged Higgs masses $M_{H^{ \pm}} \lesssim 78.9 \mathrm{GeV}[10]$.

At the LHC the dominant neutral SM (MSSM) Higgs production mechanisms (for small and moderate values of $\tan \beta$ in the MSSM) are the gluon fusion processes [11]

$$
g g \rightarrow h(h, H, A)
$$




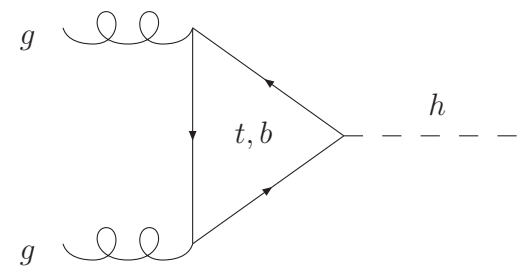

FIG. 1: Leading order contribution to the SM process $g g \rightarrow h$.

which are mediated by top and bottom loops (see Fig. 11) and for the neutral $\mathcal{C P}$-even MSSM Higgs bosons $h, H$ in addition by stop and sbottom loops with the latter contributing significantly if the squark masses are below $\sim 400 \mathrm{GeV}[12]$. At large values of $\tan \beta$ Higgs radiation off bottom quarks becomes competitive within the MSSM [13]. The NLO QCD corrections to the top and bottom loops enhance the cross sections by $50-140 \%$ for the SM Higgs boson [14, 15] and the MSSM Higgs particles for small values of $\tan \beta[14,16]$, while for large values of $\tan \beta$, where the bottom loops provide the dominant contributions due to the strongly enhanced bottom Yukawa couplings (see Fig. 22, the corrections amount to $10-60 \%$. It should be emphasized that the NLO QCD corrections are of more moderate size, if the bottom loops become dominant, while they are large in the regions of toploop dominance. The NNLO corrections are known in the heavy top mass limit, which is a valid approximation only for regions, where the top loops are dominant, i.e., for the SM and the MSSM for small values of $\tan \beta$. In this heavy top mass limit the top loop reduces to an effective $g g h$ coupling (see Fig. 3..$^{1}$ This limit is expected to provide a good approximation to the exact total cross section if $M_{h} \lesssim 2 m_{t}$. In the SM the maximal deviation from the fully massive NLO result is less than $\sim 5 \%$ in this mass range, while in the MSSM it increases to $\sim 30 \%$ for $\tan \beta \sim 5$ [13, 18], if the full mass dependence of the LO cross section is taken into account, while the $K$-factor is derived in the heavy top mass limit. Compared to the LO result, the cross section is enhanced by a factor of 1.7 to 2.3 at NNLO [19]. Moreover, the SUSY-QCD corrections have been calculated within the MSSM in the limit of heavy SUSY particle and top masses. They turn out to be large for the squark loops [12], while the genuine SUSY-QCD corrections, mediated by virtual gluino and stop exchange, are $\mathcal{O}(5 \%)$ and thus small [20]. In the gluon fusion processes the Higgs bosons are produced with vanishing transverse momenta at leading order.

Higgs boson production at finite transverse momenta requires the additional radiation of a gluon or quark in the gluon fusion process. The leading order contributions to the differential gluon fusion cross section $d \sigma / d p_{T}$ stem from diagrams as those in Fig. 4$]^{2}$ Analytical results can be found in [21].

\footnotetext{
${ }^{1}$ Note that due to the top Yukawa coupling the top contribution does not decouple for large top masses, but approaches a mass-independent value.

${ }^{2}$ In this letter we do not consider Higgs radiation off bottom quarks $g g, q \bar{q} \rightarrow b \bar{b} H$, which is of comparable size as the gluon fusion process
}

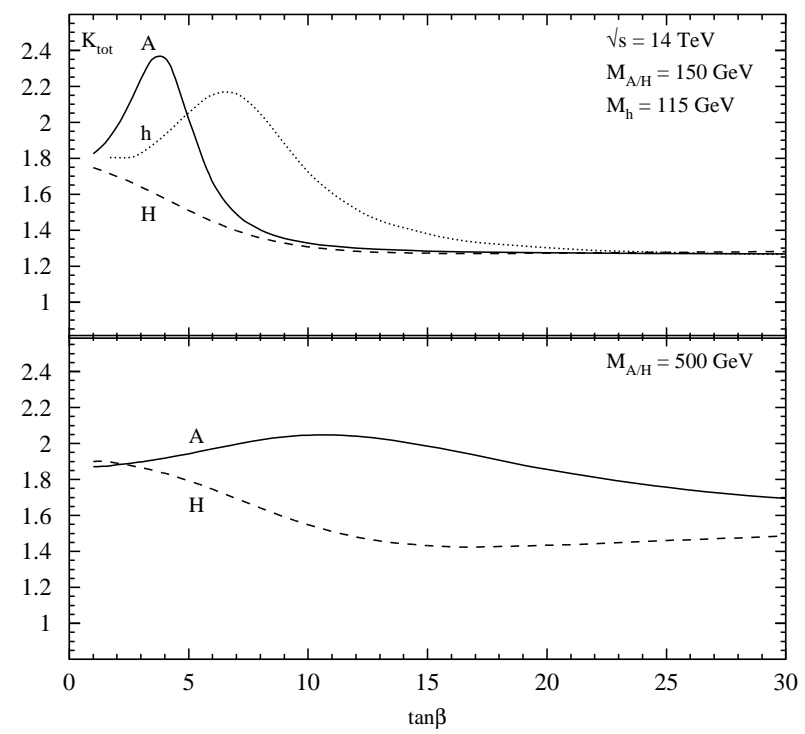

FIG. 2: Dependence of the $K$ factors for the gluon-fusion cross sections on the value of $\tan \beta$. The corresponding $K$ factors obtained by omitting the bottom loops are: $K_{h}=1.71, K_{H}=1.76\left(M_{H}=\right.$ $150 \mathrm{GeV}), K_{A}=1.78\left(M_{A}=150 \mathrm{GeV}\right), K_{H}=1.91\left(M_{H}=\right.$ $500 \mathrm{GeV})$ and $K_{A}=1.87\left(M_{A}=500 \mathrm{GeV}\right)$ independent of $\tan \beta$. CTEQ6L1 (CTEQ6M) parton densities [17] are used for the LO (NLO) cross sections with the corresponding Higgs mass as the renormalization and factorization scale.

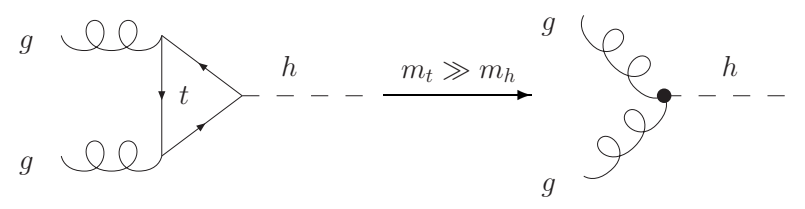

FIG. 3: Effective $g g h$ coupling in the heavy top mass limit.

NLO contributions to these expressions are again only known in the heavy top mass limit [22], which serves as an approximation for $M_{h}, p_{T} \lesssim m_{t}$. Since the bottom mass is small, this approximation is not expected to work in MSSM regions, where the bottom loop contributions are significant. At low transverse momentum $\left(p_{T} \ll M_{h}\right)$ multiple soft gluon emission spoils the validity of fixed order calculations. To obtain reliable results in this region, contributions of all orders have to be taken into account by resumming the large logarithmic terms $\ln ^{n}\left(m_{h}^{2} / p_{T}^{2}\right)$. These computations have been performed at leading logarithmic (LL), next-to-leading logarithmic (NLL) [23] and next-to-next-to-leading logarithmic (NNLL) level [24]. The NNLL calculation was performed in the heavy top mass limit. To obtain reliable predictions for the differential cross section over the whole $p_{T}$ range, the

for large values of $\tan \beta$ within the MSSM. 

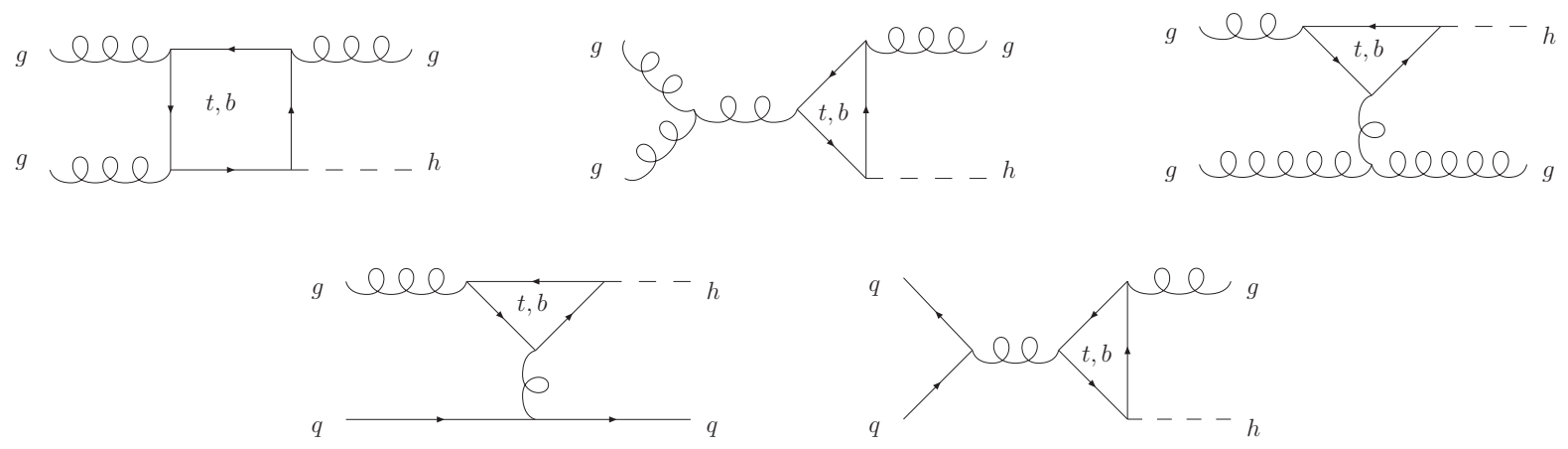

FIG. 4: LO Feynman diagrams contributing to the transverse momentum spectrum of the SM Higgs boson, mediated by Higgs couplings to gluons.

resummed results are matched to fixed order calculations at large transverse momentum. This is for example done in the HQT [25] program (for more details on the programs see below), results of which are shown in Fig.5 The heavy top mass limit is only valid as long as $p_{T} \lesssim m_{t}$. For larger values this approximation tends to overestimate the cross section [21]. This can be seen in Fig. 6 where the SM spectrum was computed with HIGLU [26] for the exact top mass and an infinite top mass.

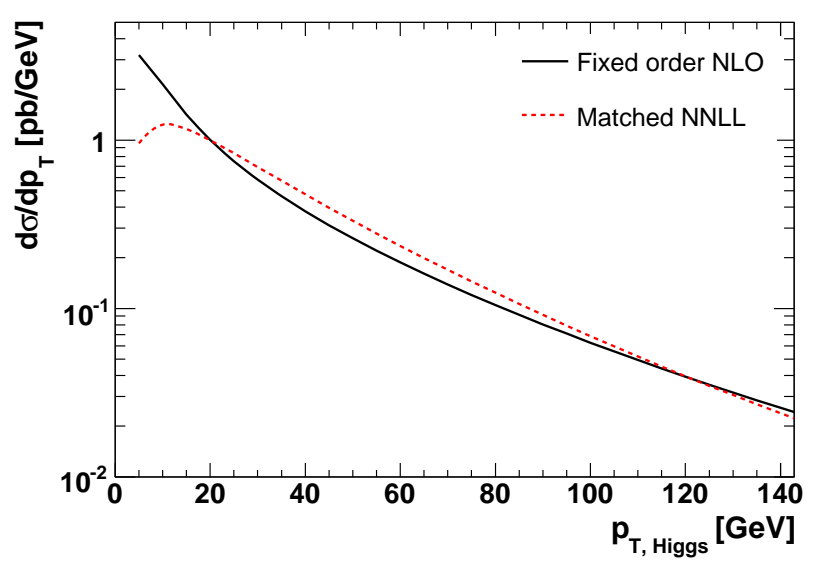

FIG. 5: Comparison of the NLO differential cross section with the NNLL result, matched to the NLO result for SM Higgs bosons with mass $M_{h}=115 \mathrm{GeV}$ in the heavy top mass limit. The renormalization and factorization scales are chosen as the transverse mass.

The development of strategies for Higgs boson searches at the LHC requires reliable estimates of the total and the differential cross section. The latter can potentially be used to discriminate signal from background, especially at large transverse momenta, where many background processes are suppressed [27]. In this letter we compare the transverse momentum distributions of the SM and MSSM Higgs bosons obtained with the programs Pythia [28], HigLu [26] and HQT [25]. In PYTHIA the large transverse momentum spectra have the same shape in the SM and the MSSM. A crosscheck of the results of PYTHIA at large transverse momenta is performed with the program HIGLU [26]. In the SM the spectrum obtained by HIGLU is softer than that of PYTHIA, because PYTHIA uses the heavy top mass approximation. The assumption in PYTHIA of similar transverse momentum shapes in the SM and the MSSM is not correct in general, especially in regions of bottom-loop dominance [29]. In some MSSM benchmark scenarios large discrepancies between the two programs are found.

\section{NUMERICAL RESULTS AND DISCUSSION}

If not mentioned otherwise, all results are given for a Higgs boson mass of $115 \mathrm{GeV}$ and a value of $\tan \beta=30$. For the numerical studies the following programs have been used:

- Pythia v6.227 [28]

The PyTHIA process $g g \rightarrow h$ uses the matrix element at order $\alpha_{s}^{2}$, i.e., the Higgs boson is produced at rest. The Higgs boson receives its transverse momentum only by initial state radiation, which is added to the hard interaction. The initial state radiation is added in such a way that the large $p_{T}$ spectrum is matched to that of the process $g g \rightarrow h g$. All calculations implemented in PYTHIA are performed in the heavy top mass limit. We have used the default scale choices.

- Higlu [26]

HIGLU is a program to compute the total Higgs production cross section via gluon fusion at NLO in the SM as well as in the MSSM. It can also be used to calculate the differential cross section at the same order in $\alpha_{s}$ (i.e. at LO), although this option is not documented. To the best of our knowledge, HIGLU is the only freely available program which allows to compute the differential spectrum in the MSSM with the full heavy quark mass dependence. The renormalization and factorization scales are chosen as the transverse mass $M_{T}=\sqrt{M_{h}^{2}+p_{T}^{2}}$. 


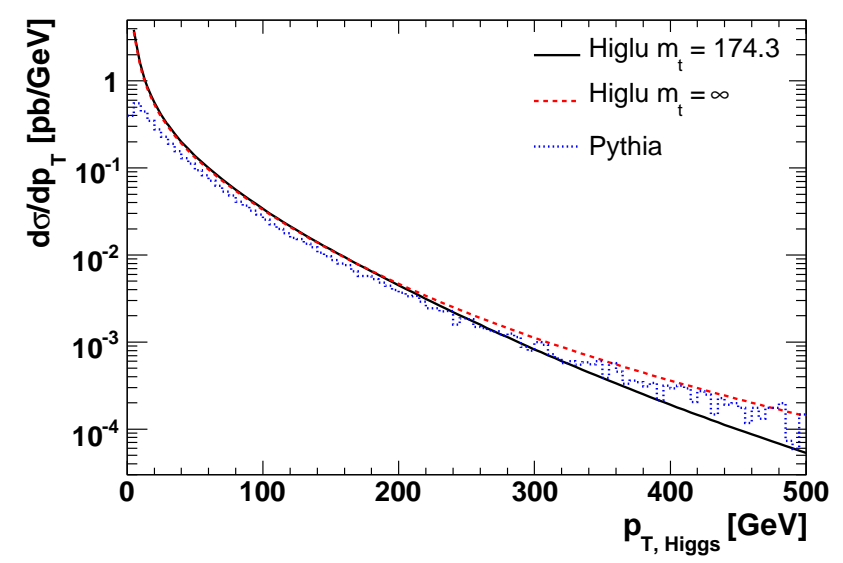

FIG. 6: Comparison of the differential spectrum in the SM generated by HigLU and PythiA for $M_{h}=115 \mathrm{GeV}$. The renormalization and factorization scales in HIGLU are chosen as the transverse mass.

\section{- HQT [25]}

HQT can be used to compute the $p_{T}$ spectrum of the Higgs boson produced in gluon fusion at LO and NLO. The spectra are calculated in the heavy top mass limit, but they can be normalized to the exact total cross sections. HQT only performs calculations in the SM. It includes a soft gluon resummation, thus providing a reliable and finite prediction in the limit of small transverse momentum in contrast to the purely perturbative result implemented in Higlu, which diverges for $p_{T} \rightarrow 0$. The renormalization and factorization scales are chosen as the transverse mass $M_{T}=\sqrt{M_{h}^{2}+p_{T}^{2}}$.

If not mentioned otherwise, CTEQ5L parton densities were used in PYTHIA, while in HIGLU and HQT CTEQ6M parton densities were used [17].

\section{A. Standard Model}

As shown in Fig. 6 good agreement between the spectra of Pythia and Higlu is obtained in the SM, if Higlu is used in the heavy top mass limit. The shapes of the two curves coincide reasonably well at large $p_{T}$. At low $p_{T}$ the HIGLU result diverges, because HIGLU does not perform any resummation. While good agreement is achieved in the heavy top mass limit, significant deviations for large values of $p_{T}$ are observed when taking into account finite quark mass effects in Higlu: the cross section at large $p_{T}$ is overestimated by the heavy top mass approximation [21] as can be inferred also from Fig. 6 Thus any analysis requiring Higgs bosons at large transverse momenta has to be adjusted accordingly.

\section{B. Minimal Supersymmetric Standard Model}

In the MSSM the comparison between PYTHIA and HIGLU was carried out for the four different benchmark scenarios

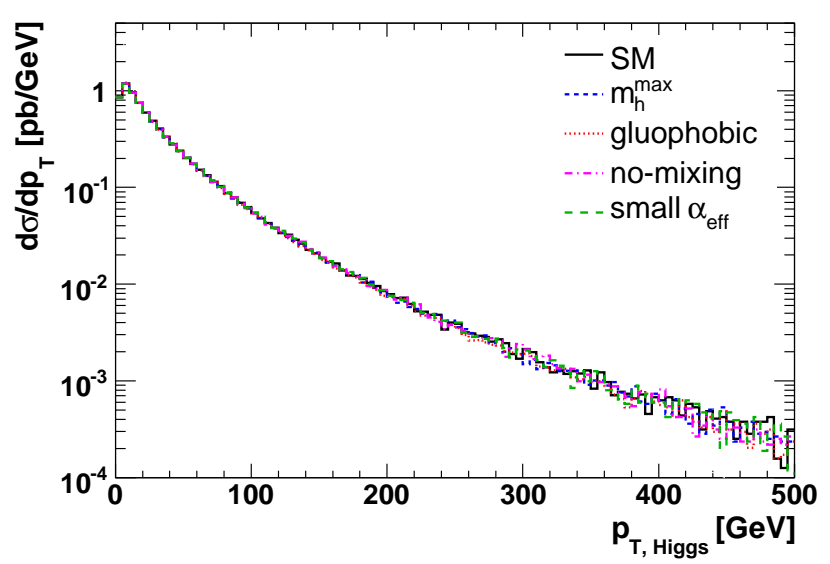

FIG. 7: PYTHIA spectra for the SM and the four benchmark scenarios scaled to the SM total cross section. The light scalar Higgs spectra are shown for $M_{h}=115 \mathrm{GeV}$.

proposed in [30], i.e., the $m_{h}^{\max }$, the no-mixing, the gluophobic Higgs and the small $\alpha_{\text {eff }}$ scenario.

In PYTHIA the shape of the spectra is the same for the SM and all MSSM scenarios, only the normalization changes. This is true even though by switching on supersymmetry in PythiA, the couplings of the quarks to the Higgs boson change compared to the SM. As in the SM, the Higgs particle is produced at rest and then initial state showers are added to the hard scattering. This shower is using a splitting kernel that is convoluted with the LO $g g \rightarrow h$ matrix element, thereby ensuring that the shower reproduces the $g g \rightarrow g h$ matrix element at large $p_{T}$ values by a proper matching procedure. This convolution of the splitting kernel with the hard interaction is performed in the heavy top mass limit in the SM and MSSM. Therefore the spectrum shapes coincide in both cases. This can be clearly seen in Fig. 7 where all spectra produced by PyThIA, scaled to the corresponding total SM cross sections, are shown.

In contrast to this, Higlu shows differently hard spectra for the benchmark scenarios, as can be inferred from Fig. 8 All spectra turn out to be softer than the SM spectrum. Taking the PYTHIA spectrum as the base of a physics analysis, the Higgs signal can be overestimated by more than an order of magnitude for $p_{T} \gtrsim 100 \mathrm{GeV}$. The softness of the spectra can be traced back to the fact, that the bottom quarks yield the main contribution to the differential cross section for large $\tan \beta$. Table $\amalg$ lists the couplings for the four benchmark scenarios. In the $m_{h}^{\max }$ scenario, the bottom loop contribution is enhanced by a factor of $1.65 \times 10^{4}$ compared to the toploop contribution alone. A lighter quark in the loop generates a softer spectrum as can be read off Fig. 9. The same effect is also visible in the spectra of the other scenarios. It is particularly large in the small $\alpha_{\text {eff }}$ scenario.

The effect of two other parameters, the Higgs mass and $\tan \beta$, on the large $p_{T}$ spectrum is shown in Figs. 10 and 11. If the mass of the light Higgs boson approaches its upper limit (which corresponds to $M_{A} \rightarrow \infty$ ), the spectrum 

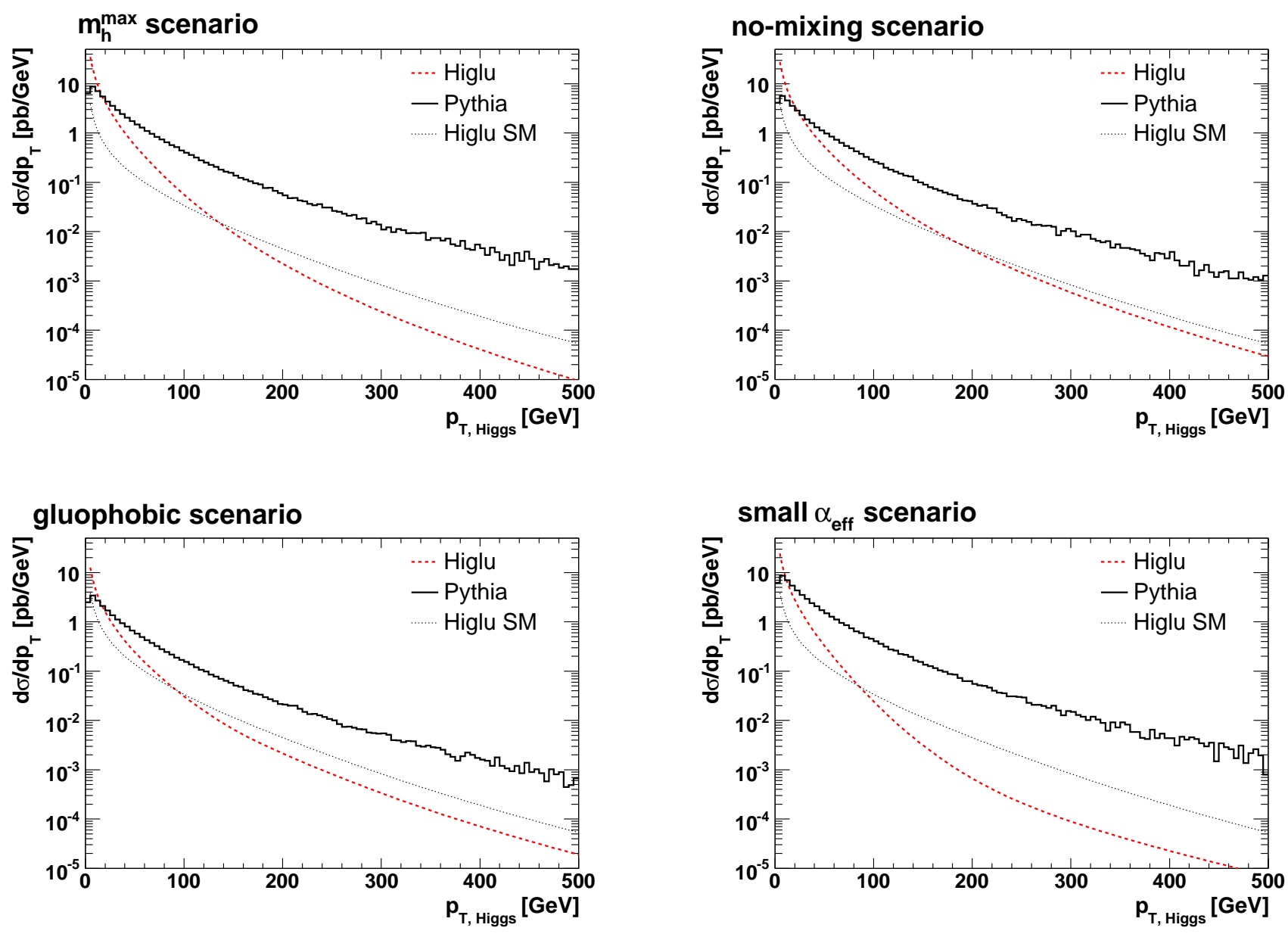

FIG. 8: Comparison of the differential spectra generated by HIGLU and PYTHIA in the four benchmark scenarios for the light scalar Higgs boson with $M_{h}=115 \mathrm{GeV}$.

TABLE II: LO cross sections and Higgs Yukawa couplings to upand down-type quarks in the four benchmark scenarios computed with HIGLU for $\tan \beta=30$ using CTEQ6L1 parton densities for the light scalar MSSM Higgs boson with $M_{h}=115 \mathrm{GeV}$.

\begin{tabular}{|l|c|c|c|c|}
\hline & $\sigma_{L O}[\mathrm{pb}]$ & $g_{d}^{h}$ & $g_{u}^{h}$ & $\left(g_{d}^{h} / g_{u}^{h}\right)^{2}$ \\
\hline $\mathrm{SM}$ & 21.8 & 1.00 & 1.00 & 1.00 \\
\hline$m_{h}^{\max }$ & 200 & 26.0 & 0.202 & $1.65 \times 10^{4}$ \\
\hline no-mixing & 158 & 24.6 & 0.544 & $2.05 \times 10^{3}$ \\
\hline gluophobic & 71.7 & 16.6 & 0.800 & $4.32 \times 10^{2}$ \\
\hline small $\alpha_{\mathrm{eff}}$ & 141 & 18.3 & -0.464 & $1.56 \times 10^{3}$ \\
\hline
\end{tabular}

becomes SM-like. The same happens if $\tan \beta$ is lowered as presented in Fig.11] since in both limits, $M_{A} \rightarrow \infty$ and small $\tan \beta$, the Higgs Yukawa couplings become similar to the SM couplings. Fig. 12 shows the variation of the spectra if the renormalization and the factorization scales are varied by a factor two around the transverse mass $M_{T}=\sqrt{M_{h}^{2}+p_{T}^{2}}$.
The transverse momentum distributions changes by $\sim \pm 40 \%$. This variation can be taken as a lower bound of the theoretical uncertainty at LO in analogy to the total inclusive gluon fusion cross section.

\section{Best estimate for the large $p_{T}$ spectrum}

The aim of this subsection is to get the best possible prediction for the differential cross section at large transverse momentum. It is clear from the preceding discussion that the following conditions have to be met:

- The full quark mass dependence has to be taken into account

- For large $\tan \beta$ the bottom quark loops must not be omitted

- The calculation has to be performed at the highest possible order in $\alpha_{s}$. 


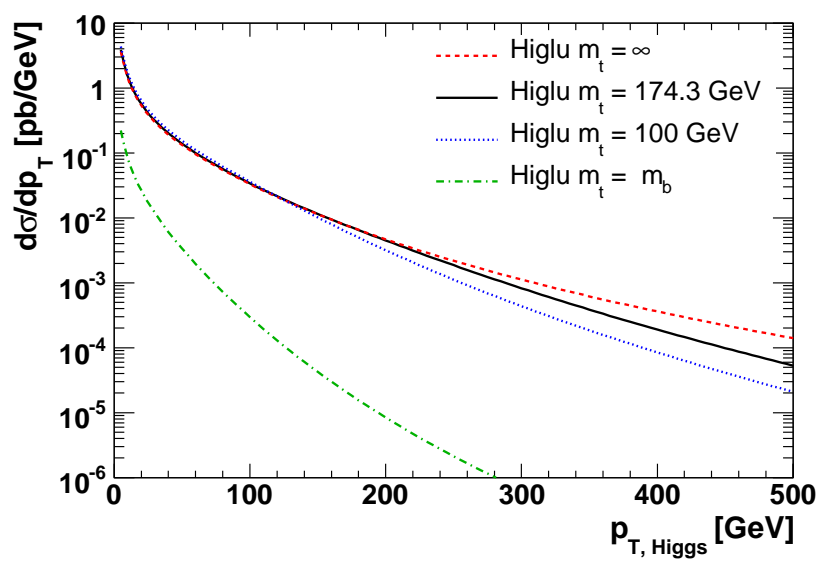

FIG. 9: Differential cross section in the SM for different top masses and $M_{h}=115 \mathrm{GeV}$.

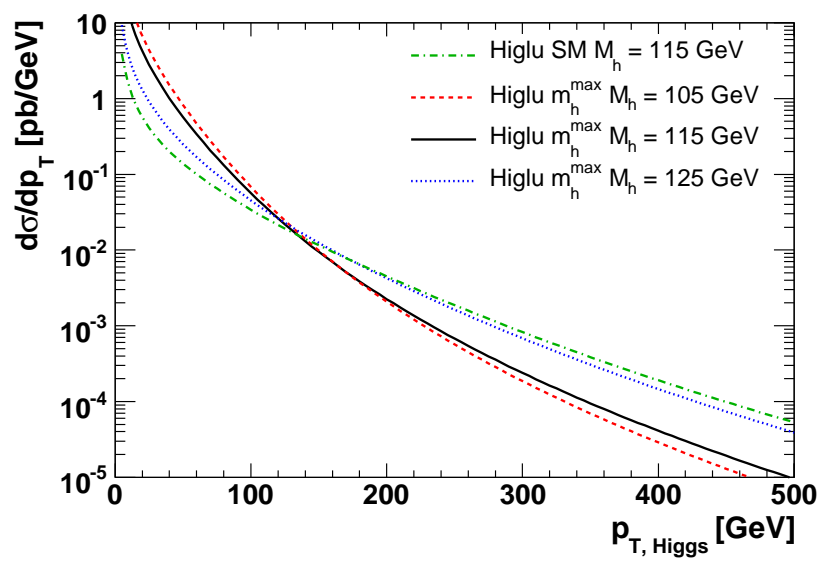

FIG. 10: Differential light scalar MSSM Higgs cross sections for different Higgs masses in the $m_{h}^{\max }$ scenario.

By using HigLu, the two first conditions are automatically met. The third one is not completely fulfilled, because HIGLU performs the calculation only at third order in the strong coupling constant. Presently there are no calculations at order $\alpha_{s}^{4}$ fulfilling the first condition, since at this order the differential cross section is only known in the heavy top mass limit. In the meantime, the following improvement to the HIGLU result is the only possibility. From the comparison of the differential cross section in the heavy top mass limit at LO and NLO a $p_{T}$ dependent $K$-factor can be extracted, which then can be applied to the exact LO result. This procedure has been carried out for the example of the $m_{h}^{\max }$ scenario. The $p_{T}$ dependent $K$-factor was computed with HQT and then applied to the LO HigLu spectrum. The result is shown in Fig. 13 The figure shows the HQT spectra at LO and NLO, from which the $K$ factor was extracted, the HIGLU result at LO as well as the scaled Higlu spectrum. For comparison the Pythia result is also given. However, it should be noted that this approximation will be valid only for small values of $\tan \beta$, where the

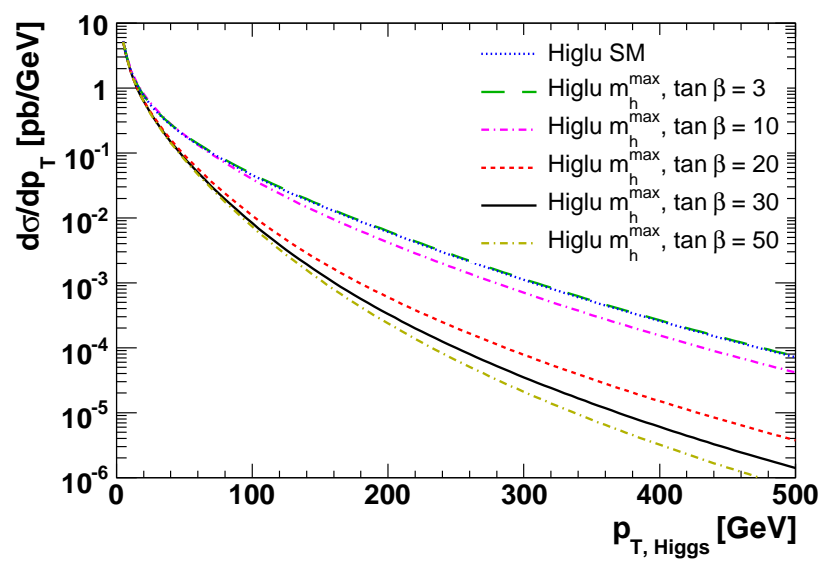

FIG. 11: Differential light scalar Higgs cross section for different values of $\tan \beta$ and $M_{h}=115 \mathrm{GeV}$. All spectra are rescaled by the ratio of the total LO SM cross section and the corresponding total LO cross section of the scenario.

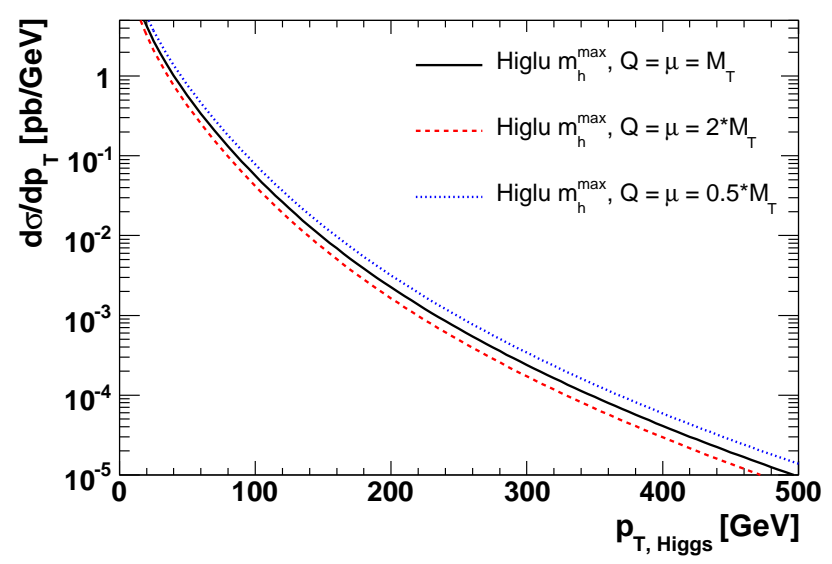

FIG. 12: Light scalar transverse momentum spectra in the $m_{h}^{\max }$ scenario for different renormalization $(\mu)$ and factorization $(Q)$ scales and $M_{h}=115 \mathrm{GeV}$.

top loops are dominant, and not too large transverse momenta, while its validity for large values of $\tan \beta$, where the bottom loops become dominant, is not clear, before a fully massive NLO calculation is available for large transverse momenta.

\section{CONCLUSION}

The large transverse momentum spectrum of the Higgs boson produced in gluon fusion was investigated with different programs. While the predictions of PYTHIA and HIGLU agree reasonably well within the SM, significant differences were found in the MSSM at large values of $\tan \beta$. Compared to Higlu, Pythia overestimates the differential cross section in some scenarios by more than one order of magnitude for $p_{T}>100 \mathrm{GeV}$. The reason for this is, that PythiA is always 


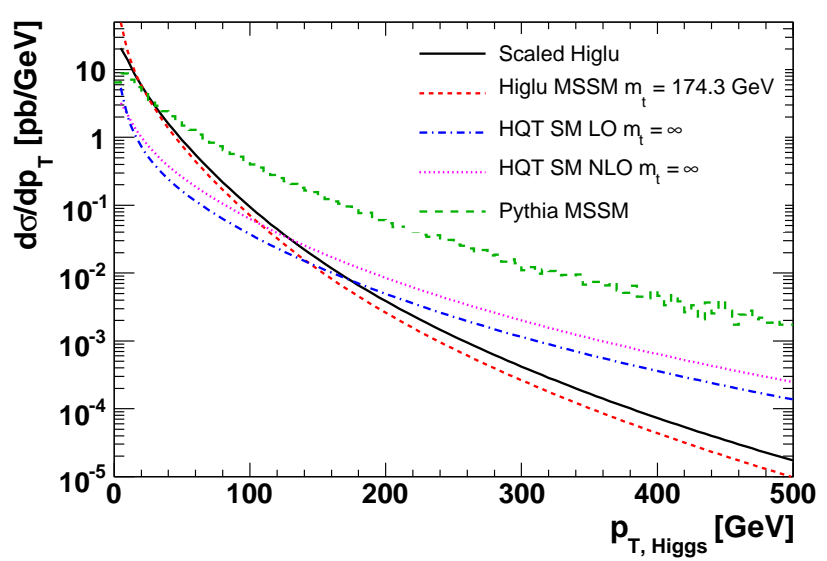

FIG. 13: Best estimate for the large transverse momentum spectrum of the light scalar Higgs boson in the $m_{h}^{\max }$ scenario for $M_{h}=$ $115 \mathrm{GeV}$. working in the heavy top mass limit, where the quark loop is replaced by an effective coupling of the two gluons to the Higgs boson. In this way, the shape of the PYTHIA spectrum is not sensitive to changes of the Higgs Yukawa couplings, which modify the relative weight of the top and bottom quark loop contributions.

\section{ACKNOWLEDGMENTS}

U.L. and P.T. acknowledge discussions with Stefano Frixione and Zoltan Kunszt. This research was partially supported by the Swiss National Science Foundation (SNF).
[1] P. W. Higgs, Phys. Lett. 12 (1964) 132, Phys. Rev. Lett. 13 (1964) 508, Phys. Rev. 145 (1966) 1156; F. Englert and R. Brout, Phys. Rev. Lett. 13 (1964) 321; G. S. Guralnik, C. R. Hagen and T. W. B. Kibble, Phys. Rev. Lett. 13 (1964) 585.

[2] N. Cabibbo, L. Maiani, G. Parisi and R. Petronzio, Nucl. Phys. B 158 (1979) 295; M. S. Chanowitz, M. A. Furman and I. Hinchliffe, Phys. Lett. B 78 (1978) 285; R. A. Flores and M. Sher, Phys. Rev. D 27 (1983) 1679; M. Lindner, Z. Phys. C 31 (1986) 295; M. Sher, Phys. Rept. 179 (1989) 273, Phys. Lett. B 317 (1993) 159 [Addendum-ibid. B 331 (1994) 448] [arXiv:hep-ph/9307342]; G. Altarelli and G. Isidori, Phys. Lett. B 337 (1994) 141; J. A. Casas, J. R. Espinosa and M. Quiros, Phys. Lett. B 342 (1995) 171 [arXiv:hep-ph/9409458]; J. R. Espinosa and M. Quiros, Phys. Lett. B 353 (1995) 257 [arXiv:hep$\mathrm{ph} / 9504241]$.

[3] A. Hasenfratz, K. Jansen, C. B. Lang, T. Neuhaus and H. Yoneyama, Phys. Lett. B 199 (1987) 531; J. Kuti, L. Lin and Y. Shen, Phys. Rev. Lett. 61 (1988) 678; M. Lüscher and P. Weisz, Nucl. Phys. B 318 (1989) 705.

[4] R. Barate et al. [LEP Working Group for Higgs boson searches], Phys. Lett. B 565 (2003) 61 [arXiv:hep-ex/0306033].

[5] For reviews on supersymmetric theories, see J. Wess and B. Zumino, Nucl. Phys. B 70 (1974) 39; P. Fayet and S. Ferrara, Phys. Rept. 32 (1977) 249; H. P. Nilles, Phys. Rept. 110 (1984) 1; H. E. Haber and G. L. Kane, Phys. Rept. 117 (1985) 75; R. Barbieri, Riv. Nuovo Cim. 11N4 (1988) 1.

[6] E. Witten, Phys. Lett. B 105 (1981) 267.

[7] S. Dimopoulos and H. Georgi, Nucl. Phys. B 193 (1981) 150; N. Sakai, Z. Phys. C 11, 153 (1981); K. Inoue, A. Kakuto, H. Komatsu and S. Takeshita, Prog. Theor. Phys. 68 (1982) 927 [Erratum-ibid. 70 (1983) 330], Prog. Theor. Phys. 71 (1984) 413.

[8] Y. Okada, M. Yamaguchi and T. Yanagida, Prog. Theor. Phys. 85 (1991) 1; H. E. Haber and R. Hempfling, Phys. Rev. Lett. 66 (1991) 1815; J. R. Ellis, G. Ridolfi and F. Zwirner, Phys. Lett. B 257 (1991) 83; R. Barbieri, M. Frigeni and F. Caravaglios, Phys. Lett. B 258 (1991) 167; A. Yamada, Phys. Lett. B 263 (1991) 233; A. Brignole, J. R. Ellis, G. Ridolfi and F. Zwirner,
Phys. Lett. B 271 (1991) 123; P. H. Chankowski, S. Pokorski and J. Rosiek, Phys. Lett. B 274 (1992) 191.

[9] J. R. Espinosa and M. Quiros, Phys. Lett. B 266 (1991) 389; R. Hempfling and A. H. Hoang, Phys. Lett. B 331 (1994) 99 [arXiv:hep-ph/9401219]; J. A. Casas, J. R. Espinosa, M. Quiros and A. Riotto, Nucl. Phys. B 436 (1995) 3 [Erratum-ibid. B 439 (1995) 466] [arXiv:hep-ph/9407389]; M. Carena, J. R. Espinosa, M. Quiros and C. E. M. Wagner, Phys. Lett. B 355 (1995) 209 [arXiv:hep-ph/9504316]; M. Carena, M. Quiros and C. E. M. Wagner, Nucl. Phys. B 461 (1996) 407 [arXiv:hepph/9508343]; S. Heinemeyer, W. Hollik and G. Weiglein, Phys. Rev. D 58 (1998) 091701 [arXiv:hep-ph/9803277], Eur. Phys. J. C 9 (1999) 343 [arXiv:hep-ph/9812472], Phys. Lett. B 440 (1998) 296 [arXiv:hep-ph/9807423], JHEP 0006 (2000) 009 [arXiv:hep-ph/9909540]; J. R. Espinosa and R. J. Zhang, Nucl. Phys. B 586 (2000) 3 [arXiv:hep-ph/0003246]; A. Brignole, G. Degrassi, P. Slavich and F. Zwirner, Nucl. Phys. B 631 (2002) 195 [arXiv:hep-ph/0112177], A. Brignole, G. Degrassi, P. Slavich and F. Zwirner, Nucl. Phys. B 643 (2002) 79 [arXiv:hep-ph/0206101]; G. Degrassi, S. Heinemeyer, W. Hollik, P. Slavich and G. Weiglein, Eur. Phys. J. C 28 (2003) 133 [arXiv:hep-ph/0212020]; S. Heinemeyer, W. Hollik, H. Rzehak and G. Weiglein, Eur. Phys. J. C 39 (2005) 465 [arXiv:hep$\mathrm{ph} / 0411114]$.

[10] [LEP Higgs Working Group], arXiv:hep-ex/0107030, arXiv:hep-ex/0602042.

[11] H. M. Georgi, S. L. Glashow, M. E. Machacek and D. V. Nanopoulos, Phys. Rev. Lett. 40 (1978) 692.

[12] S. Dawson, A. Djouadi and M. Spira, Phys. Rev. Lett. 77 (1996) 16 [arXiv:hep-ph/9603423].

[13] M. Spira, Fortsch. Phys. 46 (1998) 203 [arXiv:hepph/9705337]; A. Djouadi, arXiv:hep-ph/0503172, arXiv:hep$\mathrm{ph} / 0503173$.

[14] M. Spira, A. Djouadi, D. Graudenz and P. M. Zerwas, Nucl. Phys. B 453 (1995) 17 [arXiv:hep-ph/9504378].

[15] A. Djouadi, M. Spira and P. M. Zerwas, Phys. Lett. B 264 (1991) 440; S. Dawson, Nucl. Phys. B 359 (1991) 283. D. Graudenz, M. Spira and P. M. Zerwas, Phys. Rev. Lett. 70 (1993) 1372; S. Dawson and R. Kauffman, Phys. Rev. D 49 (1994) 
2298 [arXiv:hep-ph/9310281]

[16] M. Spira, A. Djouadi, D. Graudenz and P. M. Zerwas, Phys. Lett. B 318 (1993) 347; R. P. Kauffman and W. Schaffer, Phys. Rev. D 49 (1994) 551 [arXiv:hep-ph/9305279].

[17] J. Pumplin, D. R. Stump, J. Huston, H. L. Lai, P. Nadolsky and W. K. Tung, JHEP 0207 (2002) 012 [arXiv:hep-ph/0201195].

[18] M. Krämer, E. Laenen and M. Spira, Nucl. Phys. B 511 (1998) 523 [arXiv:hep-ph/9611272].

[19] R. V. Harlander and W. B. Kilgore, Phys. Rev. Lett. 88 (2002) 201801 [arXiv:hep-ph/0201206], JHEP 0210 (2002) 017 [arXiv:hep-ph/0208096]; C. Anastasiou and K. Melnikov, Nucl. Phys. B 646 (2002) 220 [arXiv:hep-ph/0207004], Phys. Rev. D 67 (2003) 037501 [arXiv:hep-ph/0208115]; V. Ravindran, J. Smith and W. L. van Neerven, Nucl. Phys. B 665 (2003) 325 [arXiv:hep-ph/0302135].

[20] R. V. Harlander and M. Steinhauser, Phys. Lett. B 574 (2003) 258 [arXiv:hep-ph/0307346], Phys. Rev. D 68 (2003) 111701 [arXiv:hep-ph/0308210], JHEP 0409 (2004) 066 [arXiv:hepph/0409010]; R. V. Harlander and F. Hofmann, arXiv:hep$\mathrm{ph} / 0507041$.

[21] R. K. Ellis, I. Hinchliffe, M. Soldate and J. J. van der Bij, Nucl. Phys. B 297 (1988) 221; U. Baur and E. W. N. Glover, Nucl. Phys. B 339 (1990) 38; O. Brein and W. Hollik, Phys. Rev. D 68 (2003) 095006 [arXiv:hep-ph/0305321].

[22] C. R. Schmidt, Phys. Lett. B 413 (1997) 391 [arXiv:hepph/9707448]; D. de Florian, M. Grazzini and Z. Kunszt, Phys. Rev. Lett. 82 (1999) 5209 [arXiv:hep-ph/9902483]; V. Ravindran, J. Smith and W. L. Van Neerven, Nucl. Phys. B 634 (2002) 247 [arXiv:hep-ph/0201114]; C. J. Glosser and C. R. Schmidt, JHEP 0212 (2002) 016 [arXiv:hep-ph/0209248]; C. Anastasiou, K. Melnikov and F. Petriello, Phys. Rev. Lett. 93 (2004) 262002 [arXiv:hep-ph/0409088], Nucl. Phys. B 724 (2005) 197 [arXiv:hep-ph/0501130].
[23] S. Catani, E. D'Emilio and L. Trentadue, Phys. Lett. B 211 (1988) 335; I. Hinchliffe and S. F. Novaes, Phys. Rev. D 38 (1988) 3475; R. P. Kauffman, Phys. Rev. D 44 (1991) 1415, Phys. Rev. D 45 (1992) 1512. C. Balazs and C.P. Yuan, Phys. Lett. B478 (2000) 192 [arXiv:hep-ph/0001103] ; E.L. Berger and J.W. Qiu, Phys. Rev. D67 (2003) 034026 [arXiv:hepph/0210135]; A. Kulesza and W.J. Stirling, JHEP 0312 (2003) 056 [arXiv:hep-ph/0307208]; A. Kulesza, G. Sterman and W. Vogelsang, Phys. Rev. D69 (2004) 014012 [arXiv:hepph/0309264]; A. Gawron and J. Kwiecinski, Phys. Rev. D70 (2004) 014003 [arXiv:hep-ph/0309303]; G. Watt, A.D. Martin and M.G. Ryskin, Phys. Rev. D70 (2004) 014012 [Erratumibid. D70 (2004) 079902] [arXiv:hep-ph/0309096]; A.V. Lipatov and N.P. Zotov, Eur. Phys. J. C44 (2005) 559 [arXiv:hep$\mathrm{ph} / 0501172]$.

[24] D. de Florian and M. Grazzini, Phys. Rev. Lett. 85 (2000) 4678 [arXiv:hep-ph/0008152], Nucl. Phys. B616 (2001) 247 [arXiv:hep-ph/0108273]; S. Catani, D. de Florian and M. Grazzini, Nucl. Phys. B596 (2001) 299 [arXiv:hepph/0008184]; G. Bozzi, S. Catani, D. de Florian and M. Grazzini, Phys. Lett. B564 (2003) 65 [arXiv:hep-ph/0302104].

[25] G. Bozzi, S. Catani, D. de Florian and M. Grazzini, arXiv:hep$\mathrm{ph} / 0508068$.

[26] M. Spira, arXiv:hep-ph/9510347.

[27] B. Mellado, W. Quayle and S. L. Wu, Phys. Lett. B 611, 60 (2005) [arXiv:hep-ph/0406095].

[28] T. Sjostrand, L. Lonnblad and S. Mrenna, arXiv:hep$\mathrm{ph} / 0108264$.

[29] B. Field, S. Dawson and J. Smith, Phys. Rev. D 69 (2004) 074013 [arXiv:hep-ph/0311199].

[30] M. Carena, S. Heinemeyer, C. E. M. Wagner and G. Weiglein, Eur. Phys. J. C 26 (2003) 601 [arXiv:hep-ph/0202167]. 